\title{
IoT as Non-Pharmaceutical Interventions for the Safety of Living Environments in COVID-19 Pandemic Age
}

\author{
Luciano Gamberini ${ }^{1,2 t}$, Patrik Pluchino ${ }^{1,2 * t}$, Davide Bacchin ${ }^{2}$, Andrea Zanella ${ }^{1,3}$, \\ Valeria Orso ${ }^{1,2}$, Spagnolli Anna ${ }^{1,2}$ and Daniela Mapelli ${ }^{1,2}$ \\ ${ }^{1}$ Human Inspired Technology Research Centre, University of Padova, Padova, Italy, ${ }^{2}$ Department of General Psychology, \\ University of Padova, Padova, Italy, ${ }^{3}$ Department of Information Engineering, University of Padova, Padova, Italy
}

The outbreak of the Sars-Cov-2 pandemic has changed our perception of safety in shared and public living environments including healthcare facilities, shops, schools, and enterprises. The Internet of Things (IOT) represents a suitable solution for managing anti-pandemic smart devices (e.g., UV lights, smart cameras, etc.) and increasing citizens' safety in public health crises. In this paper, we highlighted how loT technologies can be exploited as non-pharmaceutical interventions presenting the SAFE PLACE project as an implementation of this concept. The project meant to design and develop an loT system to ensure the safety and salubrity of shared environments. Advanced algorithms will be exploited to detect and classify humans' presence, gathering, usage of personal protective equipment, and considering carefully the privacy protection of individuals.

Keywords: COVID-19, Internet of Things, Non-Pharmaceutical Interventions, Safety, Artificial Intelligence

\section{INTRODUCTION}

${ }^{\dagger}$ These authors have contributed equally to this work and share first authorship

Specialty section: This article was submitted to Human-Media Interaction,

a section of the journal Frontiers in Computer Science

Received: 30 June 2021 Accepted: 26 November 2021 Published: 16 December 2021

Citation:

Gamberini L, Pluchino P, Bacchin D, Zanella A, Orso V, Anna $S$ and

Mapelli D (2021) loT as NonPharmaceutical Interventions for the Safety of Living Environments in

COVID-19 Pandemic Age.

Front. Comput. Sci. 3:733645. doi: 10.3389/fcomp.2021.733645

The world is facing one of the worst global outbreaks of recent history, the Covid-19 pandemic. To date, more than 251 million cases have been confirmed and more than 5 million deaths worldwide (WHO, 2021). Even though several vaccines have been developed and produced continuously, the available doses are not sufficient to guarantee the administration and, consequently, the safety of the entire world population. Furthermore, the order of vaccine administration will first consider the population categories at most risk (i.e., health workers, personnel of health care residences, very elderly $>80$ ) and only later the others (e.g., elderly, young adults). For this reason, it is mandatory for all the people that are still waiting for the vaccination to follow the safety regulations to contain the pandemic strictly. The various governments have implemented different safety measures since the beginning of the pandemic. Haug and colleagues (Haug et al., 2020) reported the ranking of the effectiveness of the governments' interventions considering 226 different countries (data are related to the March-April 2020 period). These are the so-called non-pharmaceutical interventions (NPIs). Findings showed that the most effective NPIs were closure or access restrictions to places (schools, shops, restaurants, hospitals, etc.) where there could be small or large gatherings, implementation of curfews, or lockdowns. A recent paper published in 2020 (Flaxman et al., 2020) reported lockdowns as the most effective NPI measure that helped reduce the spread of the SARS-CoV-2 virus. The analysis demonstrated an enormous impact of complete lockdowns all over European countries (e.g., France, Germany, Italy, Spain, etc.). A second work (Davies et al., 2020) underlined that the 
combined interventions were more effective, nevertheless, the only measure capable of bringing the $\mathrm{R}_{0}$ index close or below 1 was the complete lockdowns. In particular, the study highlighted that shorter time intervals of intense closures and the adherence to the norms of social distancing were the best solution to avoid the overwhelming of countries' health systems. Banholzer and collaborators (Banholzer et al., 2020) underlined the relevance of venue closure (i.e., environments for recreational activities, caterings industry such as pubs and restaurants, and shops except for supermarket and pharmacies) in reducing by $36 \%$ the number of new infections followed by the prohibitions of gatherings in public or private spaces (34\%) of individuals (i.e., not same household).

Nevertheless, these actions can have adverse consequences such as: decrement in the education level, isolation, and stress for the children; health issues for patients that have to postpone their treatments (e.g., chemotherapy); increment in the domestic assault; stress due to the economic impact on different categories of employers and employees that are not allowed to work. Besides, self-imposed measures such as hand washing, mask-wearing, and social distancing have been shown effective only if most individuals change their behavior in compliance with the safety measures because of risk awareness (Teslya et al., 2020). Indeed, when adopted, self-imposed actions can result in a delay of the epidemic peak, a flattening in the epidemic curve, and a reduction of the attack rate (i.e., the proportion of the population that recover/died after a severe Covid-19 infection; Teslya et al., 2020). The World Health Organization has published an updated guidance related to safety norms that have to be followed considering the use of Personal Protective Equipment (PPE) and which strategies have to be implemented in case of shortage of PPE (WHO, 2020). Social distancing, avoiding gatherings, performing hand hygiene, and ventilating indoor settings are strongly recommended practices to counteract the spreading of the pandemic, and the only viable solutions in case of lack of PPE such as face masks, goggles or face shields, gloves. Lastly, spaces health and safety are preconditions for the recovery of the economic system and the containment of more contagious variants of the Sars-Cov-2 virus. Indeed, allowing individuals to go back to work in the presence without risks and limiting the spread of the virus strains is crucial to exploit the smart technology to support people's adherence to safety measures.

\section{Risks Perception of Contagion and Compliance With Safety Measures}

The rapid spread of the Sars-Cov-2 virus has dramatically changed individuals' perception of safety in shared and public living environments. The same places that in the recent past people deemed safe are now looked at with great concern for the high possibility of contagion. This feeling applies to various living environments, including healthcare facilities, shops (e.g., supermarkets), schools, and work-related buildings. A research showed that these concerns are reported even by individuals $(60 \%$ of the respondents, more than 8,200) with a good/very good health status. They considered themselves at risk, and such circumstance affects their behavior (Motta Zanin et al., 2020).
Besides, the public perception of contagion risks influences individuals' habits, public policies to promote compliance with safety standards, and the country economy (Motta Zanin et al., 2020). Interestingly, Motta et al. showed that around 95\% of the participants considered the measures adopted by the Italian government (e.g., lockdown, curfew, social distance, PPE) not too stringent even though they modified their behaviors in everyday life completely.

Other relevant aspects have been pointed out by Siegrist and colleagues (Siegrist et al., 2021). The trust in the source (e.g., experts, governments) is a pivotal factor that influences the potential adoption of safety-related behaviors when individuals lack in knowledge and abilities to assess risks. This so-called social trust makes the people believe that the safety measures adopted by a government are not influenced by pharmaceutical or political conspiracies. Furthermore, risk perception is also affected by the severity of the symptoms related to a specific and highly contagious disease and the likelihood of being infected. An additional important individual's concern in evaluating the contagion risk was the possibility of a family member being infected.

A recent research (Barber \& Kim, 2021) on differences in worries related to the Covid-19 pandemic showed that risk perception is linked to age and gender. The risk perception is higher for the elderly (i.e., 65-81) than for young adults (i.e., 18-35) and for men compared to women. The study also highlighted behavior changes considering the variables mentioned above. Nevertheless, in contrast to all other groups, the authors underlined how older men reported the least amount of behavior changes and fewer worries. These last were linked to lower compliance with PPE (e.g., wear a mask) or general safety norms (e.g., washing hands more often, avoid public places, moments of socialization, etc.). The outcomes underline how it is crucial to understand the individuals' emotional responses to public health crises and how they may differ in specific categories of the population because they affect the potential behavioral change.

Bowman et al. (Bowman et al., 2020) pointed out that cultural differences may also play a role in the severity of the Covid-19 risks. The study highlighted that study's participants from Hong Kong (HK), one of the countries that have better contained the pandemic, were more worried than the participants from the United Kingdom (United Kingdom). These concerns were reflected in higher compliance with the safety measures. The whole $\mathrm{HK}$ sample reported wearing a mask compared to only $3 \%$ of the United Kingdom sample. Besides, social distancing was respected with a higher probability by the $\mathrm{HK}$ sample. Participants from the United Kingdom who perceived the pandemic as more threatening were more likely to comply with safety regulations. Another work described how the limitations imposed to contrast the spread of the SARS-Cov-2 may be perceived in various European countries (Meier et al., 2020). Respondents of all nationalities reported supporting the governments' safety measures (i.e., avoiding gatherings, closure of public places, frequent hand washing, wearing protective masks, etc.). Italian respondents considered the limitations imposed at a social level as more useful than Dutch and German respondents. 
Indeed, Italians implemented more often hygienic procedures, social distancing, avoidance of crowded places, and reduction in the amount of traveling. It is necessary to note that at the period of the data collection (i.e., March 2020), there were differences in the public policies among these countries, with the Italian population having the most stringent safety regulation (i.e., complete lockdown) and the higher level of contagion in Europe.

Interestingly, individuals from diverse areas inside the same country may perceive the risk differently. For instance, North Italy inhabitants stated to be more stressed and anxious than people living in South Italy (Simione and Gnagnarella, 2020).

The type of work also affects the perception of risk (Simione and Gnagnarella, 2020). Indeed, health workers declared to be more stressed due to the higher exposition to contagion risks. Findings from Dai and colleagues (Dai et al., 2020) highlighted that the main concerns of this workers category are the infection of colleagues, family members, and insufficient availability and quality of PPE. These worries may result in conditions of severe psychological distress.

A recent review (Honey-Rosés et al., 2020) focused on the perceptions related to public spaces, on their usage and for how long the impact of the Covid-19 pandemic will last, and on the potential future transformation in terms of design. The main concern is related to the general aversion towards large and crowded places, and this aspect will indeed affect how the public spaces will be designed in the near future. Urban planners and designers will have to envision public places considering the safety regulations (e.g., avoiding gatherings, keep social distancing). The contagion worries will impact the design of the indoor space (e.g., public buildings, schools, hospitals, etc.) that, for instance, will consider adding outdoor spaces to their structure and improving the systems of internal air ventilation.

Kwok and collaborators (Kwok et al., 2020) described the outcomes of a study on risk perception and adoption of preventive measures as factors that influence the behavioral responses of the Hong Kong community in the early phase of the Covid-19 outbreak. It is important to note that Hong Kong has previous experience with other recent contagious diseases (e.g., SARS). Participants' risk perception was high considering being infected ( $89 \%$ of responses between likely and very likely) and the severity of the Covid-19 symptoms if infected $(97 \%$ between severe and very severe). Self-imposed hygiene practices (e.g., masks, hands cleaning, etc.) and avoiding the travels were mostly adopted (by $>89 \%$ ) and considered effective (by $>90 \%$ ). Besides, regarding social distancing, more variable compliance was reported (33-88\%) and higher adoption by females.

A very recent review (Howard et al., 2021) focuses mainly on face masks, considering also non-medical masks as a useful tool to reduce the transmission of the viruses such as the SARS-Cov-2. Again, risk perception is reported as a crucial factor. During the outbreak in Hong Kong as the cases of Covid-19 were rapidly rising the self-reported usage of masks has increased from 74.5 to 97.5\%. In this case, the authors underline how the health authorities have to provide clear information regarding how to produce, utilize, and sanitize these masks when they can be reused. These guidelines aid to increase individuals' compliance. Furthermore, the work highlighted the importance of implementing joint strategies such as testing, tracing the infection, personal hygiene, and social distancing.

Summarizing, it is evident how compliance with the safety measures is related to the risk perception of contagion and influenced by cultural and demographical variables. Therefore, it is necessary to support compliance with such measures by exploiting a non-invasive technological approach. In the next section, the implementation of cutting-edge technologies to combat the Covid-19 pandemic is described. The progress achieved and the open challenges are explored in the domain of the Internet of Things.

\section{INTERNET OF THINGS SOLUTIONS TO FIGHT COVID-19 IN LIVING ENVIRONMENTS}

The Internet of Things (IoT) technologies allow different physical objects to communicate with each other, exchange information, control, and be controlled by other smart devices through Internet connections. In IoT systems, in particular, a large variety of sensors can be used to detect specific stimulus/ events in the environment, and communicate this information to actuators that implement actions accordingly. This powerful paradigm finds application in several fields, such as Smart Cities, Industrial 4.0, and medicine and health, in which case it is sometimes referred to as the Internet of Medical Things (IoMT; Gatouillat, 2018).

In the current historical period, with the last year characterized by the global pandemic of COVID-19, much attention has been devoted to the possible use of IoT technologies to combat it. The World Health Organization (WHO) has indicated digital technologies as an essential tool to stimulate and increase the public health response to COVID19 (WHO, 2020). So far, in the medical domain, IoT technologies are mainly applied for the early diagnosis of some diseases (Otoom et al., 2020) and real-time monitoring of infected individuals (Javaid and Khan, 2021; Dong and Yao, 2021). Besides, it is important to understand how these advanced technologies could support the compliance with the safety norms imposed by the pandemic (Kumar et al., 2020).

Concerning social distancing, it is possible to adopt shortdistance radio technologies supported by most modern smartphones, such as Bluetooth, to determine the number of people within a certain distance from the user and provide indications on the risk degree (i.e., safe, moderate, high) based on that interpersonal distance (Munir et al., 2020; Dong and Yao, 2021). Another viable solution is to utilize cost-effective wearable devices that alert users, via notification, whether another individual is physically too close considering the safety guidelines (Nadikattu et al., 2020; Nasajpour et al., 2020).

Some of these technologies respond to distance using LED warning lights EasyBand; (Tripathy et al., 2020), others more targeted to the work environment, such as Proximity and Instant Tracing which instead make use of sounds (Marín-García et al., 2020) and proved the efficacy of such feedback in reducing the 
number of close contacts among individuals. With a similar objective, another researcher proposed a real-time video processing technique to detect the violation of the social distancing norm (i.e., people distance <2 m; Rosebrock, 2020). Al-Khazraji and Nehad (2020) designed a system that, based on video processing, translated the violation of the social distancing into a visual alert (i.e., red rectangles around the faces in case of violation, green rectangles when the norm is respected) displayed on a screen placed in a potential waiting zone (the tool could be exploited at workplaces, hospitals, airports, etc.). Mbunge and colleagues (2020) described the usage of drones for monitoring the measure of social distancing.

Besides, some solutions already existed before the outbreak of the pandemic for temperature control. In the United States, the Kinsa company (Kinsa $\odot$ ) produces IoT thermometers capable to scan people and detect individuals with high body temperature. These smart devices send the collected data to an application that may alert the company on risky situations. Therefore, Kinsa can provide the authorities with interactive maps that show areas with high number of people who had fever, enabling targeted and informed interventions (McNeil, 2020). Other studies described systems for temperature control based on Artificial Intelligence (AI) manufactured by SenseTime, Megvil technology limited, and Baidu (Kummitha, 2020) to identify infected people with symptoms. Besides, a study described the usage of a smart helmet equipped with optical and thermal cameras (Mohammed et al., 2020) that detect high body temperature and send images and location (GPS) to health officer to handle the situation. Similarly, a Hong Kong company, HKBN JOS, has produced an IoT system that uses AI to record body temperature fluctuations and provide real-time observation and alarms using contactless sensors (i.e., thermal devices; HKBN 2020). These systems can be useful for controlling people's temperature, and therefore the number of infected people, also in public spaces (Ndiaye et al., 2020).

The last recommendation taken into consideration by the present work is the compliance with masks use. Iot technologies capable of detecting the absence or incorrect use (i.e., not covering completely mouth and nose) of this medical device are still rare. However, some machine learning models trained to recognize images of people with or without masks have shown how it is possible, with reasonable accuracy, to verify their presence in public and crowded environments (Chowdary et al., 2020) and hypothesize their use in smart cities that have a dense network of surveillance cameras (Rahman et al., 2020).

Finally, there are also rare examples, such as the one brought by Petrović and Kocić (2020), in which all three elements are included in a single control system. In their work, they present a system capable of monitoring temperature, social distancing, and the surgical mask's presence, which is also able to forward alarm messages to a control application.

Yet another possible utilization of IoT technologies in the fight against the spreading of the virus is related to the refreshing of the air and the sanitization of surfaces in indoor environments. A very recent research mentioned the use of IoT-based indoor sensors for air quality for the potential risk that the virus spread could be favored by the presence of pollutants in the air (Mumtaz et al., 2021). Besides, two papers (Mbunge et al., 2021; Chamola et al., 2020) have described the usage of drones with to disinfect (spray) contaminated environments. Systems for remote, automated control of building access and windows are now readily available in the marketplace. Similarly, Hearting Ventilation and Air Conditioning (HVAC) systems are widely deployed in most (public and private) buildings. Recently, some of such systems have been equipped with many sensors that continuously monitor the environmental parameters and make it possible to dynamically adapt the setting of the HVAC to guarantee maximum comfort with reduced energy expenditure. Furthermore, the system can be connected to the Internet to enable remote access and control. Finally, systems based on UVC-based disinfection, also called Ultraviolet germicidal irradiation (UVGI), have become more and more popular in the last year, thanks to their capability of killing or inactivating viral, bacterial, and fungal species employing ultraviolet (UV) light.

Today, most of such systems allow interactivity and remote control but are developed as isolated vertical solutions, incapable of horizontal communication with other systems. This hinders the realization of more complex solutions, where the functionalities of the different sub-systems are coordinated to maximize their effectiveness in preventing the spread of the viruses. In principle, the IoT technologies can enable such horizontal coordination and interactivity, even with other prevention systems considered for virus control, but to the best of our knowledge, no attempt has yet been made in this direction.

\section{A CONCEPTUAL ANALYSIS OF INTERNET OF THINGS TECHNOLOGIES AS NON-PHARMACEUTICAL INTERVENTIONS FOR SAFE AND HEALTHY LIVING ENVIRONMENTS}

Based on the information concerning the exploitation of IoT technologies as NPIs so far, it is possible to affirm that they could serve the purpose of helping in the fight against the Covid-19 pandemic. As already pointed out, the main characteristic of the studies present in literature is that they consider technologies in isolation. Dong and Yao (2021) highlighted that Internet of Things applications are relevant for sensor-based checking of Covid-19 symptoms and compliance with safety standards: monitoring of breath, blood oxygen saturation, and temperature to make diagnoses; checking the individuals' adherence to safety norms such as social distancing, gatherings, contact tracing, quarantine compliance, and proper use of PPE. In addition, IoT systems are used to predict disease outbreaks and a virus mutation (Dong and Yao, 2021) that will be the results of cloud computing, big data, and deep learning analytics.

A recent review has again highlighted the usage of IoT and IoMT (Chamola et al., 2020), particularly IoT thermometers connected to a mobile application, that allow the creation of 
maps in which outbreak regions (i.e., a high number of individuals with fevers) are highlighted. Another exploitation is telemedicine to remotely check patients' conditions which leverages on IoMT technologies. Besides, the review pointed out the usage of drones to carry out crowd surveillance in China and India or to inform citizens, using megaphones, about the safety norms that have to be followed, e.g., social distancing. In addition, drones equipped with infrared cameras to measure body temperature could serve the purpose of masses screening.

Somewhat less attention has been devoted to IoT systems that comprise different and interconnected intelligent technologies (i.e., sensors and actuators) to increase the overall safety and salubrity of entire living and working environments. Indeed, IoT integrated systems can effectively detect and/or prevent potentially dangerous situations connected with a health crisis. Considering the detection, it is possible to envisage smart cameras based on algorithms of computer vision and visual processing technique that monitor correct usage of PPE, social distancing, gatherings, and send alarms to control interfaces to alert security officers. With regards to the prevention of the spread of SARS$\mathrm{CoV}-2$, IoT technologies can operate in synergy but autonomously to sanitize indoor environments based on the current conditions, e.g., increment in the presence of pollutants that is known as favouring the spread of the virus (Mumtaz et al., 2021), and implement necessary actions. Indeed, for instance, a system for automated access (i.e., doors and windows) can guarantee air recirculation at regular temporal intervals. Furthermore, a UV-based lighting device can disinfect the surfaces/objects in the same room when a smart camera signals that the environment is empty (i.e., no people to prevent skin and eyes damage).

Nevertheless, the concept of IoT tools as NPIs faces several challenges related to its actual implementation (Kamal, Aljohani, \& Alanazi, 2020). The IoMT devices that monitor patients' conditions are required in a significant number to be effective on a large scale and the subsequent high amount of data gathered have to be managed. Concerning the transmission of data IoT devices-Cloud and vice versa, more bandwidth is necessary to avoid that delay in information transfer may lead to the death of patients. $5 \mathrm{G}$ may be the definitive solution for the IoT needs in terms of high-speed connectivity (Siriwardhana et al., 2020) without the need for WiFi or IoT gateways. IoT tools can be easily connected to $5 \mathrm{G}$ networks. Security and privacy concerns arose in IoT usage to contrast the Covid-19 pandemic (Ndiaye et al., 2020). Pertaining the security, the data collected with medical sensors have to be accurate. They have to safely reach the Cloud (cybersecurity measures for transmission and storage) for their sensitivity and confidentiality. Indeed, external hackers' attacks may steal information such as the actual position of individuals or people's images from intelligent cameras. The same worries apply to IoT devices used to increase the safety and salubrity of living spaces to avoid the incorrect activation of tools that may endanger human beings (e.g., UV lighting).

The potentiality of IoT may be boosted by improvements in Big Data analytics and Artificial Intelligence to support deep processing and the combination of heterogeneous data coming from wearable and environmental sensors that will serve as a powerful tool to face this type of health crisis. IoT devices have begun to be quite widespread however it is necessary to invest more in such technologies in the direction of creating ecosystems such as smart cities. Currently, IoT implementations adopt mainly a machine-to-machine approach and not an infrastructural perspective with more global features (Ndiaye et al., 2020). Smart cities are still a mirage for the majority of the countries around the world. In the next sections an implementation of the IoT as NPIs will be presented detailing the SAFE PLACE project.

\section{SAFE PLACE PROJECT}

The Consortium of SAFE PLACE includes Academic and Industrial partners to capitalize on their balanced mix of knowledge, skills, and expertise. Synergy will be the key factor to support the project activities effectively. The Academic partners will provide theoretical and methodological expertise in the fields of Psychology, Human-Machine Interaction, Information Engineering, Computer Science, and Cybersecurity. The Industrial partners will contribute, taking advantage of their technical competencies in the fields of advanced systems for surfaces and objects disinfection, air sanitization, ventilation, and conditioning.

The rationale behind the SAFE PLACE project is to innovate the technological devices already present on the market to make them more efficient in contrasting the spread of viruses (e.g., SARS-Cov-2), exploiting an approach based on the IoT paradigm.

\section{SAFE PLACE Project's Objectives}

Firstly, SAFE PLACE intends to design and develop advanced systems capable of exploiting the IoT technologies as NPIs to ensure people's safety in public health crises. Several smart devices will be integrated in the SAFE PLACE IoT systems.

Detection algorithms will process visual images to monitor gatherings and the correct use of PPE will be improved to avoid artifacts and misdetection. Radar technologies will also be considered to prevent privacy issues that are usually linked to normal video-cameras usage. Furthermore, Artificial Intelligence (AI) algorithms will be utilized to predict events in the identified usage scenarios. Novel solutions will be taken into account for the sanitization of air, surfaces, objects in the living environments. The project will also consider integrating commercial digital assistant devices (e.g., Google Home) in the SAFE PLACE IoT systems. Due to the specific virus typology and the ways of infection, the SAFE PLACE solutions are characterized by multiple targets: monitoring gatherings and correct usage of protective equipment, guaranteeing air salubrity, and sanitizing surfaces and objects.

\section{SAFE PLACE PROJECT'S DESCRIPTION}

SAFE PLACE comprises different work packages that will carry out specific activities, dialogue, and act in synergies. A summary 


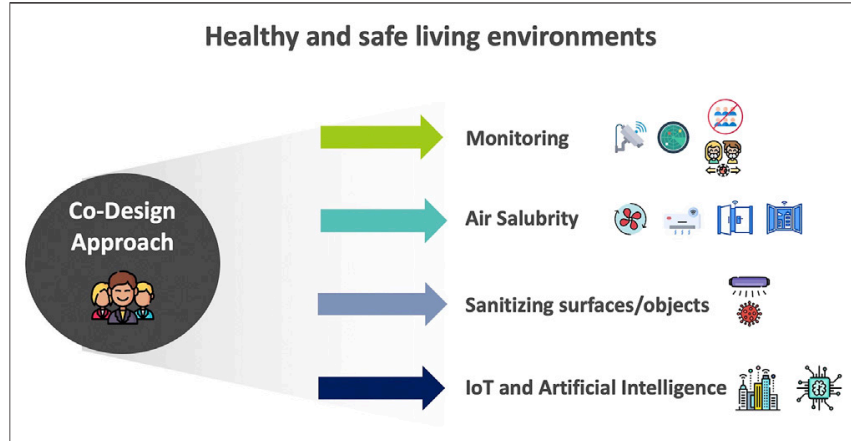

FIGURE 1 | Summary of SAFE PLACE methodological approach and hardware/software technologies.

of the project methodological approach and hardware/software technologies is depicted in Figure 1. In the following paragraphs, we illustrate the objectives and technological solutions considered.

\section{Guidelines for Technological Implementation and Co-design Approach}

The project will identify guidelines for designing healthy and safe living environments compliant to the actual safety and ethics standards, exploiting the project partners' know-how and the precious involvement of end users' groups. Besides, different usage scenarios (e.g., factories, supermarkets, classrooms) will be considered for proof-of-concept demonstrations of the capabilities of the SAFE PLACE solutions. To highlight the modularity and flexibility aspects in the design approach for the developed system, the scenarios will showcase different possible technological solutions, which include either a subset of the IoT devices and services (simple scenarios) or all of them (complex scenarios). Finally, following a co-design approach, the project will create graphical interfaces for controlling the SAFE PLACE systems. Indeed, target users and stakeholders will be involved in focus group sessions to gather their requirements, needs, expectations, and potential privacy risks related to the IoT technologies and how to eliminate or mitigate them. This methodology will permit to develop user interfaces that will be easy to use, accessible for all the users, and deliver services that will be perceived as trustworthy.

\section{Monitoring Gatherings and Correct Usage of Protective Equipment}

SAFE PLACE will develop and integrate advanced technologies for monitoring living environments, access management, and control of people flows. Industrial research will drive the development of noninvasive video systems to perform real-time control of the compliance with the standards of social distance and the usage of PPE, ensuring a low number of misdetections. An alternative noninvasive technological solution, based on radar sensing, will also be considered to assess indoor and outdoor environments' social distance. The information gathered by these and other sensors will feed computational modules to perform people tracking and control the access to public places (shops, offices, buildings) based on the level of crowding, mobility prediction, familiarity group detection, and correct use of PPE.

\section{Guaranteeing Air Salubrity of Indoor Spaces}

Environmental sensors and systems of sanitization, ventilation, and conditioning will be created to check the air quality and salubrity. In consideration of air sanitization, systems based on Non-Thermal Plasma (NTP) technologies (i.e., cold plasma) capable of reducing pollutants and microorganisms (e.g., bacteria, viruses) will be optimized and fully integrated with domotic and air conditioning systems. Furthermore, displacement ventilation systems will reduce the potential pathogenic load moving the air from areas more crowded or at risk. Lastly, doors and windows will be automatically opened at regular intervals to promote air circulation. Besides, automated control of the systems will be based on advanced algorithms that will adapt to the environmental conditions and the living space usage.

\section{Sanitizing Surfaces and Objects of Indoor Areas}

The last target of the project will pertain to prototypes design and development for the sanitization and disinfection of surfaces and objects. These prototypes will integrate ionization technologies (cold plasma) in lighting and UV-C systems that will operate following specific parameters such as the presence/absence of individuals in the monitored spaces. The devices that will exploit the bipolar ions technology will also allow the utilization when people are present in the environments. Alternatively, the UV-C systems will be employed to eliminate the pathogenic load on the surfaces and the objects when the living spaces will be empty. Besides, various lighting devices will be developed for indoor environments, following the principle of Human Centric Lighting (HCL), to improve the quality of life of individuals forced in lockdown periods or long-term care. In this WP, there will be activities related to assessing the efficacy and safety of these sanitization and disinfection systems, focusing specifically on the SARS-Cov-2 virus, involving certified laboratories identified by the consortium partners.

\section{IoT, Data Science, and Artificial Intelligence}

SAFE PLACE's actions will be devoted to the integration of the various technologies developed in the project, to enable their remote and centralized control following the IoT paradigm. Potential risks and guidelines will be identified for developing protocols to ensure the overall cybersecurity of the project's technological solutions. A user interface will be designed to permit the control of the integrated IoT systems. A web-based application will be created, interfaceable with the user interface, to visualize the data gathered from all the devices comprised in the SAFE PLACE systems. Besides, technologies and integration protocols will be built for simple and complex scenarios, exploiting a centralizer device that will permit the communication among all the IoT devices. Finally, AI algorithms will be devised to predict various aspects of the living spaces (e.g., optimization of comfort). Technical tests will be carried out in the laboratory to validate the project software and hardware 
solutions. These assessments will precede field tests. In addition, SAFE PLACE integrated systems will be able to operate also in a stand-alone form to untie their functioning from the Internet in case of connection failure.

\section{Real-World Testing and End-Users Involvement}

Finally, the in-the-field implementation of the SAFE PLACE systems will follow the validation trials. Furthermore, these tests will allow the assessments of the communication among IoT devices, the cybersecurity protocols, and the quality of the predictive AI algorithms. Security norms of personal data treatments will be considered to ensure that the developed technologies will manage, store, and treat the final users' data following the current legal regulations regarding data protection. End users will also be involved to explored usability, acceptance, and overall user experience linked to the developed systems. Various psychological and social aspects related to the introduction of SAFE PLACE systems in real-world scenarios will be explored, such as users' trust in technologies and general perception of safety in the different (i.e., simple and complex) usage scenarios.

\section{DISCUSSION}

The exploitation of IoT technologies as NPIs may serve the specific purpose of fighting the Covid-19 pandemic. Nevertheless, these systems have to adapt their functionalities, compare to the recent past, to provide a proper means to combat the widespread of SARSCov-2. SAFE PLACE considers the inclusion of suitable and feasible technological solutions in IoT integrated systems to avoid gatherings, monitor the correct usage of PPE, sanitize and sanify air, surfaces, and objects, in living environments. The aerosol transmission of SARSCov-2 is facilitated when social distancing norms are not respected, and the PPE is incorrectly utilized. Besides, it may occur under specific circumstances such as not properly ventilated indoor environments when infected individuals spend there long periods of time or when they sneeze, cough on, or touch objects or surfaces resulting in the contamination of the environment. The systems designed and developed in SAFE PLACE may be exploited to prevent the diffusion of other types of viruses transmitted in a similar fashion.

The human-centered approach in designing and developing the technologies will result in a higher degree of IoT systems acceptance, trust, and the actual usage of such technological tools. Besides, perceived security and privacy are relevant factors that influence individuals' intention to utilize services offered by smart cities and in general smart environments (Habib et al., 2020; Chin et al., 2019). The awareness of having contributed to the implementation of these systems will increase the perception of system and user' data safety. SAFE PLACE will consider several solutions to eliminate or mitigate any privacy-related issue. Cybersecurity and data privacy will be regarded seriously to protect the people that will inhabitant those living/working environments and their sensitive data. Smart cameras will not permanently record images of the end users, but rather process in real-time images to monitor the social distance and detect the presence of PPE using machine learning algorithms.
Furthermore, sensing radar will be used as an alternative way of tracking gatherings to increase the privacy protection of individuals' data. The cybersecurity risks will be comprehended and managed to increase the perception of the safety of SAFE PLACE IoT systems to prevent external cyber-attacks that could endanger the individuals (e.g., activation of UV-C lighting systems in the presence of people in the environment).

The strength of the project is to exploit synergically various IoT devices to combat and prevent the negative consequences of an health crisis. The benefits of SAFE PLACE will also reduce the impact of an outbreak on the health and economic system. The sanitization and sanification of the living environments (i.e., air, surfaces, objects), both in the presence or absence of human beings, will reduce the overall possibility of becoming infected massively.

The prototypes designed and developed within the SAFE PLACE project are conceived taking into account the potential shortcomings of IoT technologies. Simple and complex scenarios will allow the assessment of SAFE PLACE systems performance in terms of data transmission and exchange (i.e., to and from the Cloud). These preliminary analyses will shed light on the possibility of conceiving a future real-world trial in which a SAFE PLACE system will comprise all the mentioned technologies at once.

Regarding communication across the IoT devices, the project will consider different protocols (e.g., WiFi, MQTT, ZigBee) and the inclusion of voice assistants (i.e., Amazon Alexa, Google Home). Indeed, one of the project aims is to integrate devices through commercial IoT devices to maximize the market attractiveness and competitiveness of the SAFE PLACE systems.

Artificial intelligence and deep learning will be exploited for visual processing and computer vision to refine the ability of IoT tools to detect and/or prevent dangerous situations linked to noncompliance with safety standards. By doing so, the performance of SAFE PLACE systems will improve itself over time based on the growing amount of data collected regarding both people's behaviors and specific events in the monitored environments.

Individuals will be safer to go to work and the support to follow the correct procedure of PPE usage, provided by the IoT systems will reduce the spread of the virus.

\section{AUTHOR CONTRIBUTIONS}

Conceptualization, PP, VO, AZ, AS, DM, and LG; resources, LG; writing-original draft preparation, $\mathrm{PP}, \mathrm{DB}, \mathrm{VO}$; writing - review and editing, PP, DB, AZ, AS, DM, LG; visualization, PP; supervision, LG; project administration, LG; funding acquisition, LG. All authors have read and agreed to be accountable for the content of the work.

\section{FUNDING}

This work was supported by the POR FESR 2014-2020 Work Program of the Veneto Region (Action 1.1.4) through the project No. 10288513 titled"' SAFE PLACE. Sistemi IoT per ambienti di vita salubri e sicuri”. 


\section{REFERENCES}

Al-Khazraji, A., and Nehad, A. E. (2020). "November)Smart Monitoring System for Physical Distancing," in 2020 Second International Sustainability and Resilience Conference: Technology and Innovation in Building Designs (IEEE), 1-3. doi:10.1109/IEEECONF51154.2020.931993251154

Banholzer, N., van Weenen, E., Kratzwald, B., Seeliger, A., Tschernutter, D., Bottrighi, P., et al. (2020). Impact of Non-pharmaceutical Interventions on Documented Cases of COVID-19. MedRxiv.

Barber, S. J., and Kim, H. (2021). COVID-19 Worries and Behavior Changes in Older and Younger Men and Women. The Journals Gerontol. Ser. B 76 (2), e17-e23. doi:10.1093/geronb/gbaa068

Bowman, L., Kwok, K., Redd, R., Yi, Y., Ward, H., Wei, W., Atchison, C., and Wong, S. (2020). Public Perceptions and Preventive Behaviours during the Early Phase of the COVID-19 Pandemic: a Comparative Study between Hong Kong and the United Kingdom. MedRxiv. doi:10.1101/2020.08.06.20169409

Chamola, V., Hassija, V., Gupta, V., and Guizani, M. (2020). A Comprehensive Review of the COVID-19 Pandemic and the Role of IoT, Drones, AI, Blockchain, and 5G in Managing its Impact. Ieee access 8, 90225-90265. doi:10.1109/access.2020.2992341

Chin, J., Callaghan, V., and Allouch, S. B. (2019). The Internet-Of-Things: Reflections on the Past, Present and Future from a User-Centered and Smart Environment Perspective. Ais 11 (1), 45-69. doi:10.3233/AIS-180506

Dai, Y., Hu, G., Xiong, H., Qiu, H., and Yuan, X. (2020). Psychological Impact of the Coronavirus Disease 2019 (COVID-19) Outbreak on Healthcare Workers in China. MedRxiv. doi:10.1101/2020.03.03.20030874

Dong, Y., and Yao, Y.-D. (2021). IoT Platform for COVID-19 Prevention and Control: A Survey. IEEE Access 9, 49929-49941. doi:10.1109/ access.2021.3068276

Flaxman, S., Mishra, S., Gandy, A., Unwin, H. J. T., Mellan, T. A., Coupland, H., et al. (2020). Estimating the Effects of Non-pharmaceutical Interventions on COVID-19 in Europe. Nature 584 (7820), 257-261. doi:10.1038/s41586-020-2405-7

Habib, A., Alsmadi, D., and Prybutok, V. R. (2020). Factors that Determine Residents' Acceptance of Smart City Technologies. Behav. Inf. Technology 39 (6), 610-623. doi:10.1080/0144929X.2019.1693629

Haug, N., Geyrhofer, L., Londei, A., Dervic, E., Desvars-Larrive, A., Loreto, V., et al. (2020). Ranking the Effectiveness of Worldwide COVID-19 Government Interventions. Nat. Hum. Behav. 4 (12), 1303-1312. doi:10.1038/s41562-020-01009-0

Honey-Rosés, J., Anguelovski, I., Chireh, V. K., Daher, C., Konijnendijk van den Bosch, C., Litt, J. S., et al. (2020). The Impact of COVID-19 on Public Space: an Early Review of the Emerging Questions - Design, Perceptions and Inequities. Cities \& Health, 1-17. doi:10.1080/23748834.2020.1780074

Howard, J., Huang, A., Li, Z., Tufekci, Z., Zdimal, V., van der Westhuizen, H.-M., et al. (2021). An Evidence Review of Face Masks against COVID-19. Proc. Natl. Acad. Sci. USA 118 (4), e2014564118. doi:10.1073/pnas.2014564118

Javaid, M., and Khan, I. H. (2021). Internet of Things (IoT) Enabled Healthcare Helps to Take the Challenges of COVID-19 Pandemic. J. Oral Biol. Craniofac. Res. 11, 209-214. doi:10.1016/j.jobcr.2021.01.015

Jignesh Chowdary, G., Punn, N. S., Sonbhadra, S. K., and Agarwal, S. (2020). "December)Face Mask Detection Using Transfer Learning of Inceptionv3," in International Conference on Big Data Analytics (Springer, Cham), 81-90. doi:10.1007/978-3-030-66665-1_6

Kamal, M., Aljohani, A., and Alanazi, E. (2020). IoT Meets COVID-19: Status, Challenges, and Opportunities. arXiv preprint arXiv:2007.12268.

Kumar, K., Kumar, N., and Shah, R. (2020). Role of IoT to Avoid Spreading of COVID-19. Int. J. Intell. Networks 1, 32-35. doi:10.1016/j.ijin.2020.05.002

Kummitha, R. K. R. (2020). Smart Technologies for Fighting Pandemics: The Techno- and Human- Driven Approaches in Controlling the Virus Transmission. Government Inf. Q. 37, 101481. doi:10.1016/j.giq.2020.101481

Kwok, K. O., Li, K.-K., Hin Chan, H. H., Yi, Y. Y., Tang, A., Wei, W. I., et al. (2020). Community Responses during the Early Phase of the COVID-19 Epidemic in Hong Kong: Risk Perception, Information Exposure and Preventive Measures. MedRxiv. doi:10.1101/2020.02.26.20028217

Marín-García, D., Moyano-Campos, J. J., and Bienvenido-Huertas, J. D. (2020). Distances of Transmission Risk of COVID-19 inside Dwellings and Evaluation of the Effectiveness of Reciprocal Proximity Warning Sounds. Indoor air 31 (2), 335-347. doi:10.1111/ina.12738
Mbunge, E., Akinnuwesi, B., Fashoto, S. G., Metfula, A. S., and Mashwama, P. (2021). A Critical Review of Emerging Technologies for Tackling COVID -19 Pandemic. Hum. Behav Emerg Tech 3 (1), 25-39. doi:10.1002/hbe2.237

McNeil, D. (2020). Can Smart Thermometers Track the Spread of the Coronavirus. New York, NY: The New York Times. Available at: https://www.nytimes.com/ 2020/03/18/health/coronavirusfever-thermometer\%s.html.

Meier, K., Glatz, T., Guijt, M. C., Piccininni, M., Van Der Meulen, M., Atmar, K., et al. (2020). Public Perspectives on Protective Measures during the COVID-19 Pandemic in the Netherlands, Germany and Italy: A Survey Study. PloS one 15 (8), e0236917. doi:10.1371/journal.pone.0236917

Mohammed, M. N., Syamsudin, H., Al-Zubaidi, S., Aks, R. R., and Yusuf, E. (2020). Novel COVID-19 Detection and Diagnosis System Using IOT Based Smart Helmet. Int. J. Psychosocial Rehabil. 24 (7), 2296-2303.

Motta Zanin, G., Gentile, E., Parisi, A., and Spasiano, D. (2020). A Preliminary Evaluation of the Public Risk Perception Related to the COVID-19 Health Emergency in Italy. Ijerph 17 (9), 30241-30320. doi:10.3390/ijerph17093024

Mumtaz, R., Zaidi, S. M. H., Shakir, M. Z., Shafi, U., Malik, M. M., Haque, A., et al. (2021). Internet of Things (IoT) Based Indoor Air Quality Sensing and Predictive Analytic-A COVID-19 Perspective. Electronics 10 (2), 184. doi:10.3390/electronics10020184

Munir, M. S., Abedin, S. F., and Hong, C. S. (2020). A Risk-Sensitive Social Distance Recommendation System via Bluetooth towards the COVID-19 Private Safety. Proc. Korean Soc. Inf. Sci., 1028-1030.

Nadikattu, R. R., Mohammad, S. M., and Whig, D. (2020). Novel Economical Social Distancing Smart Device for COVID19. Int. J. Electr. Eng. Technology 11 (4). doi:10.34218/ijeet.11.4.2020.023

Nasajpour, M., Pouriyeh, S., Parizi, R. M., Dorodchi, M., Valero, M., and Arabnia, H. R. (2020). Internet of Things for Current COVID-19 and Future Pandemics: An Exploratory Study. J. Healthc. Inform. Res. 4, 325-364. doi:10.1007/s41666020-00080-6

Ndiaye, M., Oyewobi, S. S., Abu-Mahfouz, A. M., Hancke, G. P., Kurien, A. M., and Djouani, K. (2020). IoT in the Wake of COVID-19: A Survey on Contributions, Challenges and Evolution. IEEE Access 8, 186821-186839. doi:10.1109/ ACCESS.2020.3030090

Otoom, M., Otoum, N., Alzubaidi, M. A., Etoom, Y., and Banihani, R. (2020). An IoT-Based Framework for Early Identification and Monitoring of COVID-19 Cases. Biomed. Signal Process. Control. 62, 102149. doi:10.1016/ j.bspc. 2020.102149

Petrović, N., and Kocić, Đ. (2020). IoT-based System for COVID-19 Indoor Safety Monitoring. preprint), IcETRAN, 1-6.

Rahman, M. M., Manik, M. M. H., Islam, M. M., Mahmud, S., and Kim, J.-H. (2020). An Automated System to Limit COVID-19 Using Facial Mask Detection in Smart City Network. 2020 IEEE International IOT, Electronics and Mechatronics Conference (IEMTRONICS). IEEE, 1-5. doi:10.1109/ IEMTRONICS51293.2020.9216386

Rosebrock, A. (2020). OpenCV Social Distancing Detector. Availableat https:// www.pyimagesearch.com/2020/06/01/opencv-social-distancing-detector/.

Siegrist, M., Luchsinger, L., and Bearth, A. (2021). The Impact of Trust and Risk Perception on the Acceptance of Measures to Reduce COVID-19 Cases. Risk Anal. 41 (0), 787-800. doi:10.1111/risa.13675

Simione, L., and Gnagnarella, C. (2020). Differences between Health Workers and General Population in Risk Perception, Behaviors, and Psychological Distress Related to COVID-19 Spread in Italy. Front. Psychol. 11, 2166. doi:10.3389/ fpsyg.2020.02166

Siriwardhana, Y., De Alwis, C., Gur, G., Ylianttila, M., and Liyanage, M. (2020). The Fight against the COVID-19 Pandemic with 5G Technologies. IEEE Eng. Manag. Rev. 48 (3), 72-84. doi:10.1109/emr.2020.3017451

Teslya, A., Pham, T. M., Godijk, N. G., Kretzschmar, M. E., Bootsma, M. C. J., and Rozhnova, G. (2020). Impact of Self-Imposed Prevention Measures and ShortTerm Government-Imposed Social Distancing on Mitigating and Delaying a COVID-19 Epidemic: A Modelling Study. Plos Med. 17 (7), e1003166. doi:10.1371/journal.pmed.1003166

Tripathy, A. K., Mohapatra, A. G., Mohanty, S. P., Kougianos, E., Joshi, A. M., and Das, G. (2020). EasyBand: a Wearable for Safety-Aware Mobility during Pandemic Outbreak. IEEE Consumer Electron. Mag. 9 (5), 57-61. doi:10.1109/MCE.2020.2992034

World Health Organization (2020). Rational Use of Personal Protective Equipment for COVID-19 and Considerations during Severe Shortages: Interim Guidance. 
New York, NY: World Health Organization. No. WHO/2019-nCoV/ IPC_PPE_use/2020.4 23 December 2020.

World Health Organization (2021). World Health Organization. Availableat: https://covid19.who.int (Accessed November 12, 2021).

Conflict of Interest: The authors declare that the research was conducted in the absence of any commercial or financial relationships that could be construed as a potential conflict of interest.

Publisher's Note: All claims expressed in this article are solely those of the authors and do not necessarily represent those of their affiliated organizations, or those of the publisher, the editors, and the reviewers. Any product that may be evaluated in this article, or claim that may be made by its manufacturer, is not guaranteed or endorsed by the publisher.

Copyright $\odot 2021$ Gamberini, Pluchino, Bacchin, Zanella, Orso, Anna and Mapelli. This is an open-access article distributed under the terms of the Creative Commons Attribution License (CC BY). The use, distribution or reproduction in other forums is permitted, provided the original author(s) and the copyright owner(s) are credited and that the original publication in this journal is cited, in accordance with accepted academic practice. No use, distribution or reproduction is permitted which does not comply with these terms. 